\title{
La dimension urbaine de la politique européenne de cohésion et la construction métropolitaine : le cas de l'Ile-de-France
}

Brice Lamenie

\section{(2) OpenEdition \\ Journals}

Édition électronique

URL : https://journals.openedition.org/cdg/5347

DOI : $10.4000 /$ cdg. 5347

ISSN : 2107-7266

Éditeur

UMR 245 - CESSMA

Référence électronique

Brice Lamenie, "La dimension urbaine de la politique européenne de cohésion et la construction métropolitaine : le cas de l'lle-de-France », Carnets de géographes [En ligne], 14 | 2020, mis en ligne le 30 novembre 2020, consulté le 22 mai 2021. URL : http://journals.openedition.org/cdg/5347 ; DOI : https://doi.org/10.4000/cdg.5347

Ce document a été généré automatiquement le 22 mai 2021.

\section{(c) $(1)$}

La revue Carnets de géographes est mise à disposition selon les termes de la Licence Creative Commons Attribution - Pas d'Utilisation Commerciale - Pas de Modification 4.0 International. 


\title{
La dimension urbaine de la politique européenne de cohésion et la construction métropolitaine : le cas de l'Ile-de-France
}

\author{
Brice Lamenie
}

\section{Contexte, problématisation et méthodologie}

1 Cette thèse apporte un éclairage sur les mécanismes de réception territorialisés des politiques européennes. Son ambition était de contribuer à la réflexion sur les effets de l'intervention de l'Union européenne sur la gouvernance territoriale et urbaine. Ce travail s'inscrit dans la lignée des travaux sur les dynamiques de l'européanisation au miroir du local (Muller, 1997 ; Pasquier et Weisbein, 2004), s'intéressant à la façon dont l'Union européenne se confronte aux intérêts locaux. Il aborde l'européanisation en considérant les logiques ascendantes (uploading) et descendantes (downloading) comme les deux faces d'un même processus «Two-way process» (Börzel, 2002; Bocquillon, Evrard, 2016).

2 Je me suis référé à la dimension urbaine de la politique européenne de cohésion, qui est une des entrées des politiques de l'Union Européenne (UE) sur les territoires. En France, ce volet de la politique de cohésion, mis en œuvre par les intercommunalités, cible prioritairement les quartiers en difficulté. Il est piloté par les conseils régionaux depuis le transfert, en 2014, d'une grande partie des fonds européens en provenance de l'Etat. En Ile-de-France, 10 intercommunalités étaient concernées au cours de la période de programmation 2007-2013 et 15 (dont la ville de Paris) lors de la programmation 2014-2020. 
Figure 1. Les territoires du volet urbain de la politique de cohésion en lle-de-France

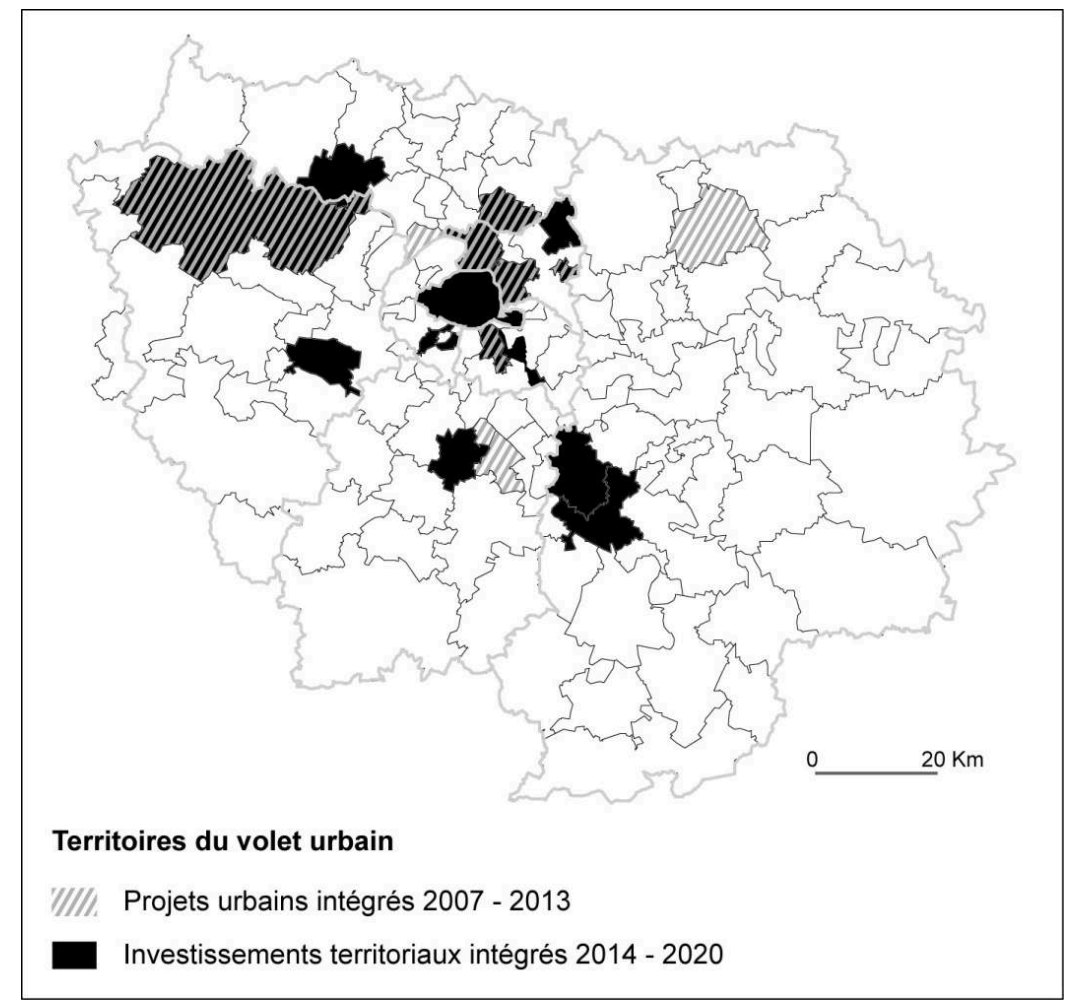

Source : Brice Lamenie et Liliane Lizzi UMR Géographie-Cités d'après préfecture de région et conseil régional lle-de-France

4 L'analyse de la gouvernance de la dimension urbaine de la politique européenne de cohésion en Ile-de-France, au cours de ces deux dernières programmations permet d'attirer l'attention sur la spécificité de cette région dans l'organisation territoriale française. L'île-de-France est un terrain d'étude privilégié des enjeux liés aux recompositions territoriales en cours, suite à la dernière réforme (2014-2015). L'échelon métropolitain, absent des débats en début de programmation, émerge ici au sein d'un territoire, certes bien maillé, mais qui n'offre pas une vision unifiée en termes de gouvernance, et dont le pilotage est toujours assuré par l'État en dépit des phases successives de décentralisation depuis 1982 (Estèbe, Le Galès ; 2003 ; Subra, 2012).

Ce travail s'est appuyé très largement sur mon expérience professionnelle en collectivité en cours de thèse. Dans ce cadre, j'ai eu l'opportunité d'accéder aux instances de concertation (groupes de travail, comités de programmation des fonds européens). J'ai pu y collecter de la documentation grise et réaliser une série d'observations. En dehors, j'ai conduit une cinquantaine d'entretiens semi-directifs auprès d'acteurs chargés de l'élaboration et de la mise en œuvre de la politique de cohésion en Ile-de-France (État, Région, départements, intercommunalités et communes concernées), au sein des institutions européennes et, dans un souci de mise en perspective avec une région métropolitaine partageant des similitudes, à Londres. Dans la capitale britannique, j'ai réalisé des entretiens avec des acteurs de la Métropole du Grand Londres (Greater London Authority), des Boroughs de Hackney et Lewisham, et auprès de bénéficiaires des fonds européens.

6 Mon hypothèse de départ concernait l'existence d'une scène européenne de la construction institutionnelle de la métropole, comme espace de négociations des 
enjeux propres à chacune des échelles engagées. Pour la valider, j'ai d'abord cherché à établir le système de relations dans lequel sont impliqués les différents niveaux de collectivités et l'État. Puis, je me suis penché sur le fonctionnement des collectivités pour comprendre comment elles mobilisent la politique de cohésion afin de concevoir leur propre stratégie européenne.

\section{Principaux résultats}

7 Plusieurs résultats ont pu être formulés à l'issue de ce travail. Grâce au transfert des fonds européens, le conseil régional dispose d'un nouveau levier qu'il peut adosser à ses politiques. Le programme opérationnel régional, qui consigne les orientations de la politique de cohésion, lui permet également d'élargir ses champs d'intervention, en incluant, par exemple, le décrochage scolaire qui relève des compétences de l'Etat.

Dans le contexte francilien, l'UE n'est pas une sphère autonome de l'action publique territoriale. Les formes de la mobilisation locale vis-à-vis de la politique de cohésion consacrent les coopérations territoriales, engagées avant la création de la Métropole du Grand Paris, et les stratégies locales élaborées en lien avec les dispositifs régionaux de l'État et de la Région.

De même, la Métropole ne se construit pas autour d'une stratégie unifiée et partagée face à l'UE. La Région Île-de-France partage une stratégie différente de celle de la ville de Paris, qui elle-même diverge de celle de la Métropole du Grand Paris. Au niveau intercommunal, l'implication dans les programmes communautaires demeure très variable selon les territoires.

Actuellement, s'il existe une scène européenne de la construction métropolitaine en Île-de-France, impulsé par les acteurs locaux, elle se conçoit autour des réseaux d'acteurs impliqués dans la conduite du volet urbain de la politique européenne de cohésion. Dans une certaine mesure, la politique de cohésion a pu constituer un outil d'amorçage au bénéfice de la coopération territoriale en Ile-de-France. Elle a participé à la rencontre des acteurs locaux et à la formalisation de stratégies de développement territorial. Elle a permis aussi d'engager des formes souples de coopération interterritoriale à travers le portage de projets miroirs ou la création d'instances de dialogue partagées.

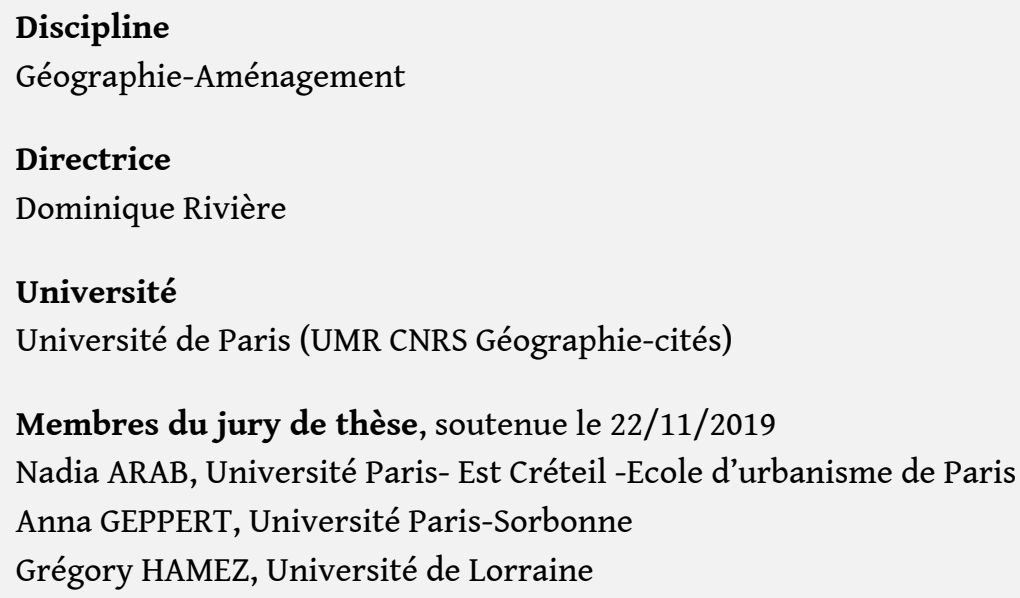


Dominique RIVIERE, Université de Paris

Frédéric SANTAMARIA, Université de Grenoble-Alpes

Situation professionnelle à l'issue de la thèse

Chargé d'enseignements Université de Paris

Chercheur associé à l'UMR CNRS Géographie-cités

Courriel de l'auteur

lamenie_brice [at] yahoo.fr

\section{BIBLIOGRAPHIE}

BOCQUILLON P., EVRARD A. (2016), « Rattraper ou devancer l'Europe : Politiques françaises des énergies renouvelables et dynamiques d'européanisation ", Politique européenne, no. 52, pp. 32-à 56.

BÖRZEL T. (2002), « Pace-setting, foot-dragging, and fence-sitting : member state responses to europeanization », Journal of Common Market Studies, vol. 40, no. 2, p. 193-214.

ESTEBE P., LE GALES P. (2003), « La métropole parisienne : à la recherche du pilote ? », Revue française d'administration publique, vol. 107 no. 3, pp. 345-356.

MULLER P. (1997), «L'européanisation des politiques publiques », Politiques et management public, vol. 15, no 1, pp. 3-9.

PASQUIER R., WEISBEIN J. (2004), « L'Europe au microscope du local. Manifeste pour une sociologie politique de l'intégration communautaire. ", Politique européenne, vol. 1, no. 12, pp. 5-21.

SUBRA P. (2012), Le Grand Paris, Géopolitique d'une ville mondiale, Paris, Armand Colin.

INDEX

Thèmes : Carnets de soutenances 